\title{
Esophageal Basaloid Carcinoma
}

National Cancer Institute

\section{Source}

National Cancer Institute. Esophageal Basaloid Carcinoma. NCI Thesaurus. Code C7032.

A rare morphologic variant of esophageal squamous cell carcinoma. Histologically, it is composed of closely packed cells with hyperchromatic nuclei and scant basophilic cytoplasm. It has a similar prognosis to the conventional squamous cell carcinoma of the esophagus. (WHO) 